\title{
Contagion Analysis in the Banking Sector
}

\author{
S., DAROLLES ${ }^{(1)}$, S., DUBECQ ${ }^{(2)}$ and C., GOURIEROUX ${ }^{(3)}$
}

(October, 2013)

Preliminary version - Do not distribute

This Working Paper should not be reported as representing the views of the European Central Bank (ECB) or those of the Autorité de Contrôle Prudentiel et de Révolution (ACPR). The views expressed are those of the authors and do not necessarily reflect those of the ECB or of the ACPR.

\footnotetext{
${ }^{1}$ University Paris-Dauphine and CREST. serge.darolles@dauphine.fr

${ }^{2}$ European Central Bank. Simon.Dubecq@ecb.int

${ }^{3}$ CREST and University of Toronto. christian.gourieroux@ensae.fr
} 


\section{Abstract \\ Contagion Analysis in the Banking Sector}

This paper analyses how an external adverse shock will impact the financial situations of banks and insurance companies and how it will diffuse among these companies. In particular we explain how to disentangle the direct and indirect (contagion) effects of such a shock, how to exhibit the contagion network and how to detect the "superspreaders", i.e. the most important firms involved in the contagion process. This method is applied to a network of 8 large European banks in order to analyze whether the revealed interconnections within these banks differ depending on the underlying measure of banks' financial positions, namely their market capitalization, the price of the CDS contract written on their default and their book value.

Keywords: Contagion, Systemic Risk, Default Dependence, Credit Default Swaps, Canonical Correlation.

EFM Classification codes: 510, 520. 


\section{Introduction}

The new regulation on financial stability lists global systematically important financial institutions (G-SIFIs), which have to comply with specific regulatory requirements. One of the criteria for a bank to be identified as systematically important is its interconnectedness. In this respect our paper studies how an external adverse shock will impact the financial situations of the banks and insurance companies and how it will diffuse among these companies. In particular we explain how to disentangle the direct and indirect (contagion) effects of such a shock, how to exhibit the contagion network and how to detect the most important firms involved in the contagion process, that are the "superspreaders", especially the institutions, which are "too interconnected to fail".

Such an analysis depends crucially on the way the financial situation of a bank or an insurance company is measured. In the academic literature as well as in the approaches used to implement Basel regulation, this financial situation is analyzed in three alternative ways:

i) by considering the balance sheets of the firms and typically their (accounting) equity value defined as the difference between their assets and their liabilities in the firm's book;

ii) by analyzing their market values when they are quoted on a stock exchange;

iii) by focusing on the information on their potential defaults by means of the prices of their issued bonds, or of the associated Credit Default Swaps (CDS) ${ }^{4}$.

There exist links between these three approaches which have been first mentioned by Merton [Merton (1974)]. Let us denote $V_{t}=A_{t}-L_{t}$ the value of the firm at date $t$, where $A_{t}$ and $L_{t}$ are the asset and liability components of the balance

\footnotetext{
${ }^{4}$ The bond and credit derivative markets are highly dependent, since there exists a relation between the CDS spread and the spread of a bond, at least theoretically [see e.g. the analysis for European Sovereign debts in Delatte et al. (2012)].
} 
sheet, respectively. Then, under standard regularity conditions, the firm's market capitalization (or market value) at date $t$ is equal to:

$$
\operatorname{Cap}_{t}=\frac{1}{1+r_{t}} \mathbb{E}_{t}^{\mathbb{Q}}\left(V_{t+1}^{+}\right)=\frac{1}{1+r_{t}} \mathbb{E}_{t}^{\mathbb{Q}}\left[\left(A_{t+1}-L_{t+1}\right)^{+}\right], \forall t
$$

and the price of the short-term digital CDS which protects the CDS's holder from a firm's default over the next period of time is:

$$
C D S_{t}=\frac{1}{1+r_{t}} \mathbb{Q}_{t}\left(V_{t+1}<0\right)=\frac{1}{1+r_{t}} \mathbb{Q}_{t}\left(A_{t+1}<L_{t+1}\right), \forall t
$$

where $r_{t}$ is the short term riskfree rate, $\mathbb{Q}_{t}$ the risk-neutral distribution conditional on the information available at date $t, V^{+}=\max (V, 0)$, and $\mathbb{E}_{t}^{\mathbb{Q}}$ the expectation with respect to $\mathbb{Q}_{t}$. Equation (1.1) corresponds to the Merton's interpretation of a stock as a European call written on the asset component with a strike equal to the liability.

The capitalization, the book value of the firm and a CDS price are different notions as are also their rates of change. Typically the stock return $\frac{\operatorname{Cap}_{t+1}}{\operatorname{Cap}_{t}}-1$ differs from $\frac{V_{t+1}}{V_{t}}-$ 1 , and from $\frac{\mathrm{CDS}_{t+1}}{\mathrm{CDS}_{t}}-1$. To better understand these differences, let us assume a zero riskfree rate $r_{t}=0$, a risk-neutral distribution equal to the historical distribution and a value of the firm which can be decomposed as:

$$
V_{t+1}=\mu_{t}+\sigma_{t} u_{t+1}, \forall t
$$

where the shocks $u_{t+1}$ are independent standard normal variables $\forall t, \mu_{t}, \sigma_{t}$ the conditional mean and standard error, respectively. Then we get:

$$
\begin{aligned}
\frac{V_{t+1}}{V_{t}} & =[\mathrm{TBC}], \\
\frac{\mathrm{Cap}_{t+1}}{\mathrm{Cap}_{t}} & =[\mathrm{TBC}], \\
\frac{\mathrm{CDS}_{t+1}}{\mathrm{CDS}_{t}} & =[\mathrm{TBC}],
\end{aligned}
$$


where $\psi(u)=u \Phi(u)+\varphi(u)$, and $\varphi, \Phi$ are the probability and cumulative density functions of the standard normal, respectively. This simplified example shows clearly that the three notions capture the first and second-order conditional moments of the underlying risks in the balance sheet in different ways. The CDS price depends on the mean/standard error ratio only after a nonlinear transform, the capitalization on mean and standard error in a more complicated way, while the ex-post observation $V_{t+1}$ involves an additional stochastic shock $u_{t+1}$.

The aim of our paper is to compare the analysis of contagion based on the rate of changes on the value of the firm, on the capitalization and on the transformed (or standardized) CDS price $\Phi^{-1}\left(C D S_{t}\right)$, respectively. In Section 2 we introduce a linear dynamic model for a joint analysis of rates of changes for several firms. This model allows both for common unobservable exogenous shocks and for contagion phenomena. We study the second-order properties of such models. We develop in Section 3 an approach to estimate the number of underlying factors and the sensitivities of the financial situations of the firms to these factors. The approach is applied to a set of banks and to the three alternative measures of their financial situations. Section 4 describes different estimation methods of the parameters of interest including the contagion matrix. We compare the structure of the estimated contagion matrices according to the selected measures of financial situations. Section 5 concludes. Proofs and additional informations are gathered in Appendices.

\section{Dynamic factor model with dynamic frailty and contagion}

\subsection{The model}

Let us consider $n$ institutions, and stack the variables of interest [which can be either the rate of change in the book value, in the market capitalization, or in the standardized digital CDS prices of the credit institutions on period $(t-1, t)$ ] in a

\footnotetext{
${ }^{5}$ To ensure a transformed CDS price in the same domain of variation as the capitalization.
} 
n-dimensional vector $Y_{t}$. These variables satisfy the following dynamic factor model:

$$
\left\{\begin{array}{l}
Y_{t}=B F_{t}+C Y_{t-1}+u_{t}, \\
F_{t}=\Phi F_{t-1}+v_{t},
\end{array}\right.
$$

where the error terms $u_{t}, v_{t}$ are zero-mean, serially independent, with second-order moments :

$$
\mathbb{V}\left(u_{t}\right)=\Sigma, \mathbb{V}\left(v_{t}\right)=\Omega, \operatorname{Cov}\left(u_{t}, v_{t}\right)=0
$$

$F_{t}$ gives the values at date $t$ of $K$ unobservable factors, called dynamic frailties in the credit risk literature [see Duffie, Eckner, Horel, Saita (2009)]. The second subsystem of (2.1) and the noncorrelation condition (2.2) means that these factors (frailties) have an exogenous dynamics. The first subsystem shows that the variables of interest (either the rate of changes on the value of the firm, or on the capitalization, or on the transformed CDS price) are dynamically dependent through the effects of the common factors, measured by the $(n, K)$ matrix of beta coefficients, and through the effects of their lagged value, measured by the $(n, n)$ contagion matrix $C$. For expository purpose we have not introduced an intercept in the return (resp. changes in standardized price) equation. Indeed, whenever the process $\left(Y_{t}\right)$ is stationary, this intercept can be set to zero by considering the demeaned return (or demeaned change in price) $Y_{t}-\bar{Y}$.

We can find in the literature special cases of dynamic model (2.1), with either common factor only, or contagion only. We review in Table A.1 in Appendix 1 this literature distinguishing the model with factor only, either observed, or unobserved, and the models with contagion only. We also mention if they are applied to balance sheet data, stock data, bond data, or CDS data.

In this paper, we focus on the contagion matrix $C$ for different indicators of institutions' financial soundness (i.e. either its book value, its market value, or its CDS standardized price). We prefer to let unobservable the factors $F_{t}$ to prevent a bad selection of observable factors from contaminating the estimation of matrix $C$. 
Moreover, model (2.1) considers jointly the return and exogenous factor dynamics. This approach is particularly useful for the purpose of predictions, risk measures, or stress-tests. Indeed, it takes into account the uncertainty on the future values of the factors as well as the implied dependence between future returns (resp. changes in standardized prices) due to the factors ${ }^{6}$.

As usual the latent factors are defined up to an invertible linear transformation. Thus we introduce identification restrictions on the parameters.

Proposition 1 (Identification restrictions): Without loss of generality, we can assume either IR1: $\Omega=I d$, or IR2: $B^{\prime} B=I d$, if $B$ is with full column rank $K$.

Proof : The first identification restriction is obtained by the change of factor $F \rightarrow$ $Q F$ with $Q=\Omega^{-1 / 2}$, where $\Omega^{-1 / 2}$ denotes the inverse of the square root of the symmetric positive definite matrix $\Omega$. The second identification restriction with $Q=\left(B^{\prime} B\right)^{-1 / 2}$.

The second-identification restriction IR 2 shows that what matters is not the matrix $B$ itself, but more the vector space spanned by $B F_{t}$ when $F_{t}$ varies. The condition $B^{\prime} B=I d$ means that the columns of $B$ can be chosen as an orthonormal basis of this vector space with dimension $K$.

Finally, a significant part of the literature on contagion considers that the returns are uncorrelated with their own past and analyzes the structure of conditional heteroscedasticity. By model (2.1), we follow the opposite approach focusing on the expression of expected returns and assuming conditional homoscedasticity. In particular, we expect $\mathrm{B}$ and $\mathrm{C}$ to be significant. The main reason for this significance is the specificity of financial institutions. The asset component of their balance sheet

\footnotetext{
${ }^{6}$ The literature on factor models considers frequently observable factors, such as for instance a market return, the inflation rate ... (see Table A.1 in Appendix 1). Observable factors are often treated in a misleading way, when we are interested in prediction, risk measures, or stresstests. Theoretically, the prediction of these future values requires a dynamic model (as the second subsystem of (2.1)), but very often in practice they are predicted by deterministic scenarios.
} 
can be seen as portfolios of basic assets. Loosely speaking we have:

$$
A_{t}=\sum_{j=1}^{J} a_{j t} p_{j t}
$$

where $j$ is the index of the basic asset, $p_{j t}$ its price, and $a_{j t}$ its quantity. If the portfolio allocation is crystallized $a_{j t}=a_{j}, \forall t$, and if the returns on the basic asset are i.i.d, the return on $\sum_{j=1}^{J} a_{j} p_{j, t}$ will also be close to i.i.d. But the role of a financial institution is to update frequently its portfolio with observed prices, that is, the allocations $a_{j, t}$ are functions of current and past prices. This allocation adjustment will destroy the i.i.d. property of the changes in asset value, in particular their serial independence, even if this property is satisfied on the basic assets.

\section{$2.2 \quad$ State space representation}

Model (2.1)-(2.2) is a Vector AutoRegressive (VAR) model with partial observability. This model admits a state space representation. More precisely let us introduce the state variable $Z_{t}=\left(Y_{t}^{\prime}, F_{t}^{\prime}\right)^{\prime}$. We have:

\section{State equation:}

$$
Z_{t}=\Psi Z_{t-1}+w_{t}
$$

where

$$
\Psi=\left(\begin{array}{cc}
C & B \Phi \\
0 & \Phi
\end{array}\right), w_{t}=\left(\begin{array}{c}
u_{t}+B v_{t} \\
v_{t}
\end{array}\right), V w_{t}=\left(\begin{array}{ll}
\Sigma+B \Omega B^{\prime} & B \Omega \\
\Omega B^{\prime} & \Omega
\end{array}\right) .
$$

\section{Measurement equation:}

$$
Y_{t}=(I d, 0) Z_{t}
$$

Thus the linear Kalman filter can be used to compute recursively the linear predictions of future values of $Y$, the filtered values of the unobservable factors as well as 
the values of a Gaussian pseudo-likelihood function [see e.g. Reinsel (1993), Section , Gourieroux, Monfort (1997), Section ].

\subsection{Second-order properties}

By considering the VAR dynamic of state process $\left(Z_{t}\right)$, we can deduce its first and second-order moments (see Appendix 2). In particular, the autocovariance of the observable process depends on the lag in the way given in Proposition 2.

Proposition 2: The second-order properties of $\left(Y_{t}\right)$ are the following:

i) The autocovariance function $\Gamma_{Y}(h)$ of the observable process $Y$ is equal to:

$$
\Gamma_{Y}(h)=\operatorname{Cov}\left(Y_{t}, Y_{t-h}\right)=C^{h} \Gamma_{Y}(0)+D_{h} \operatorname{Cov}(F, Y), h \geq 0,
$$

where $D_{h}=\sum_{k=0}^{h-1}\left(C^{k} B \Phi^{h-k}\right)=C D_{h-1}+B \Phi^{h}$.

ii) The unconditional covariance between the observable process $Y$ and the frailty $\operatorname{Cov}(Y, F)$ is solution of:

$$
\operatorname{Cov}(Y, F)=C \operatorname{Cov}(Y, F) \Phi^{\prime}+B \Phi V(F) \Phi^{\prime}+B \Omega
$$

By Proposition 2.i), we get the standard component $C^{h} \Gamma_{Y}(0)$ for a VAR dynamic for the observable process $\left(Y_{t}\right)$ with autoregressive matrix $C$ plus the term $D_{h} \operatorname{Cov}(F, Y)$ due to the unobservable factor. $D_{h}$ is a rather complicated function of $h$. Indeed, the unobservability of $F$ implies a $V A R(\infty)$ dynamic with an infinite autoregressive lag, when process $\left(Y_{t}\right)$ is considered alone.

The unconditional covariance between $Y$ and $F$ is solution of a system of Riccati equations, which in general has to be solved numerically. As seen below, the different formulas are greatly simplified for a single factor model. 


\subsection{The single factor model}

Let us consider the case $K=1$ and the model:

$$
\left\{\begin{array}{l}
Y_{t}=B F_{t}+C Y_{t-1}+u_{t} \\
F_{t}=\varphi F_{t-1}+v_{t}
\end{array}\right.
$$

where

$$
V\left(u_{t}\right)=\Sigma, V\left(v_{t}\right)=\omega, \operatorname{Cov}\left(u_{t}, v_{t}\right)=0 .
$$

We get:

$$
\Psi^{h}=\left(\begin{array}{cc}
C^{h} & d_{h} \\
& \\
0 & \varphi^{h}
\end{array}\right)
$$

where

$$
d_{h}=\sum_{k=0}^{h-1}\left(C^{k} \beta \varphi^{h-k}\right)=\varphi^{h}\left[\sum_{k=0}^{h-1}(C / \varphi)^{k}\right] B .
$$

We deduce:

$$
d_{h}=\varphi^{h}(I d-C / \varphi)^{-1}\left(I d-C^{h} / \varphi^{h}\right) B .
$$

Then the expressions of the unconditional variances and covariance become:

$$
\begin{gathered}
V(F)=\frac{w}{1-\varphi^{2}}, \\
\operatorname{Cov}(Y, F)=(I d-\varphi C)^{-1} \frac{w}{1-\varphi^{2}} \beta, \\
V(Y)=\Gamma_{Y}(0)=\sum_{h=0}^{\infty} C^{h} \Delta C^{h^{\prime}} .
\end{gathered}
$$




\subsection{Comparing datasets with different frequencies}

The indicators of banks' financial soundness we aim at investigating may not necessarily be available at the same frequency. In particular, indicators based on accounting data are accessible at a lower frequency than the market based ones. To facilitate the comparison with the analysis on market data, we can consider the daily model (2.1) for the returns on book values. Due to the state space representation, the model's form remain unchanged at lower frequency:

$$
\left\{\begin{array}{l}
Y_{t}=D_{h} F_{t}+C^{h} Y_{t-h}+u_{t, h}, \\
F_{t}=\Phi^{h} F_{t-h}+v_{t, h}
\end{array}\right.
$$

say, with $h=60$ opening days at quarterly frequency.

\section{Estimation of the number of factors and of their effects}

In a static factor model without contagion $(C=0)$ and no dynamic $(\varphi=0)$, the number of factors, the factors and the beta coefficients are usually obtained by applying a principal component analysis, based on the spectral decomposition of the historical variance-covariance matrix of the observable variables.

We use a similar approach valid for model (2.1) with both contagion and factor dynamics. The new approach allows for the estimation of the number of factors and of the betas, but does not provide approximations of the factor themselves. This approach is based on the notion of directions immunized to shocks on the factors.

As mentioned in Section 2.1, the possible effects of the factors on the vector of returns (resp. changes in standardized price) belong to the space $\mathcal{E}(B)$ generated by the columns of matrix $B$. 
Let us now consider an element $\gamma$ of the space orthogonal to $\mathcal{E}(B)$. We get:

$$
\gamma^{\prime} Y_{t}=\gamma^{\prime} B F_{t}+\gamma^{\prime} C Y_{t-1}+\gamma^{\prime} u_{t}=\gamma^{\prime} C Y_{t-1}+\gamma^{\prime} u_{t}
$$

These directions define portfolio allocations, which are immunized against the latent common factors. By considering these directions, we also eliminate the effect on $Y_{t}$ of lagged values of $Y$ with a lag larger or equal to 2.

Let us now consider the linear regression of $\gamma^{\prime} Y_{t}$ on both $Y_{t-1}, Y_{t-2}$ :

$$
\gamma^{\prime} Y_{t}=C_{1}(\gamma) Y_{t-1}+C_{2}(\gamma) Y_{t-2}+\tilde{u}_{t}, \text { say }
$$

The theoretical regression coefficients are:

$$
\begin{aligned}
{\left[C_{1}(\gamma), C_{2}(\gamma)\right] } & =\operatorname{Cov}\left[\gamma Y_{t},\left(\begin{array}{c}
Y_{t-1} \\
Y_{t-2}
\end{array}\right)\right]\left[V\left(\begin{array}{c}
Y_{t-1} \\
Y_{t-2}
\end{array}\right)\right]^{-1} \\
& =\gamma\left[\Gamma_{Y}(1), \Gamma_{Y}(2)\right]\left[\begin{array}{cc}
\Gamma_{Y}(0) & \Gamma_{Y}(1) \\
\Gamma_{Y}(1)^{\prime} & \Gamma_{Y}(0)
\end{array}\right]^{-1}
\end{aligned}
$$

We deduce that:

$$
C_{2}(\gamma) \equiv \gamma C_{2}
$$

where $C_{2}=\left[\Gamma_{Y}(1)-\Gamma_{Y}(2) \Gamma_{Y}(0)^{-1} \Gamma_{Y}^{\prime}(1)\right]\left[\Gamma_{Y}(0)-\Gamma_{Y}(1) \Gamma_{Y}(0)^{-1} \Gamma_{Y}(1)^{\prime}\right]^{-1}$, by inverting by blocks.

The matrix $C_{2}$ is a multivariate partial autocovariance of order 2 [see e.g. Ramsey (1974)]. Let us now introduce the additional identification condition below.

Identification Restriction IR3: Rank $C_{2}=K$.

Then we get the following property. 
Proposition 3: Under the identification restriction IR3 the immunizing vectors $\gamma \in \mathcal{E}(B)^{\perp}$ are the vectors of the kernel of $C_{2}$.

Corollary 1: For any positive definite $(n, n)$ matrix $\mathrm{S}$, the matrix $C_{2} S C_{2}^{\prime}$ admits exactly $n-K$ eigenvalues equal to zero. A basis of orthonormal eigenvectors of this matrix is such that the eigenvectors associated with the non zero eigenvalues are the column vectors of a $B$ matrix, which satisfies $B B^{\prime}=I d$, and the eigenvectors associated with the zero eigenvalues are immunizing vectors.

We deduce from Corollary 1 consistent estimation methods for the number of factors $K$, a matrix $B$ of beta coefficients and a basis of immunizing vectors. They follow the steps below.

step 1 : Compute the estimated multivariate partial autocovariance of order 2 by substituting in the expression of $C_{2}$ the autocovariances by their sample counterparts $\hat{C}_{2}$.

step 2 : Select a metric $S$ and perform the spectral decomposition of $\hat{C}_{2} S \hat{C}_{2}^{\prime}$, with the eigenvalues written in a decreasing order.

step 3 : Estimate $K$ by the first-order $\hat{K}$ for which the eigenvalues of $\hat{C}_{2} S \hat{C}_{2}^{\prime}$ are non significant.

step 4 : Estimate $B$ by considering the first $\hat{K}$ orthonormal eigenvectors as columns of $\hat{B}$.

step 5 : Estimate a basis of immunizing vectors by considering the next $n-\hat{K}$ orthonormal eigenvectors.

Such an approach is the analogue for Vector Autoregressive process of the analysis of codependence directions introduced for Vector Moving Average processes by Gourieroux, Peaucelle (1983), (1993) .

There exist several possible choices of the metric $S$.

\footnotetext{
${ }^{7}$ See also Kugler, Schwendeuer (1992), Engle, Kozicki (1993), Vahid, Engle (1997).
} 
i) When:

$$
S=\hat{\Gamma}_{Y}(0)-\hat{\Gamma}_{Y}(1) \hat{\Gamma}_{Y}(0)^{-1} \hat{\Gamma}_{Y}(1)^{\prime}
$$

we get:

$$
\begin{aligned}
\hat{C}_{2} S \hat{C}_{2}^{\prime}= & {\left[\hat{\Gamma}_{Y}(1)-\hat{\Gamma}_{Y}(2) \hat{\Gamma}_{Y}(0)^{-1} \hat{\Gamma}_{Y}^{\prime}(1)\right]\left[\hat{\Gamma}_{Y}(0)-\hat{\Gamma}_{Y}(1) \hat{\Gamma}_{Y}(0)^{-1} \hat{\Gamma}_{Y}(1)^{\prime}\right]^{-1} } \\
& {\left[\hat{\Gamma}_{Y}(1)^{\prime}-\hat{\Gamma}_{Y}(1) \hat{\Gamma}_{Y}(0)^{-1} \hat{\Gamma}_{Y}(2)^{\prime}\right] }
\end{aligned}
$$

with a simple interpretation of the test statistics as a multivariate estimated partial autocorrelation.

ii) It is also possible to choose $S$ in order to get some optimality properties of some test statistics based on $\hat{C}_{2} S \hat{C}_{2}^{\prime}$, such as the sum of its eigenvalues $\xi_{1}=\operatorname{Tr}\left(\hat{C}_{2} S \hat{C}_{2}^{\prime}\right)$, or $\xi_{3}=$ largest eigenvalue of $\hat{C}_{2} S \hat{C}_{2}^{\prime}$ [see Gourieroux, Monfort, Renault (1999), for a discussion].

When the identification restriction IR3 is not satisfied, the approach above can be extended by increasing the lag in autoregression (3.1) defining the immunizing directions. For a given lag $p$, we will estimate the partial autocovariance:

$\left[C_{1}(\gamma), C_{2}(\gamma), \ldots, C_{p}(\gamma)\right]=\gamma\left[\Gamma_{Y}(1), \ldots, \Gamma_{Y}(p)\right]\left[\begin{array}{ccc}\Gamma_{Y}(0) & \Gamma_{Y}(1) & \Gamma_{Y}(p-1) \\ \vdots & & \Gamma_{Y}(1) \\ \Gamma_{Y}(p-1) & \ldots & \Gamma_{Y}(0)\end{array}\right]^{-1}$

and look for its kernel.

\section{Estimation of the parameters for a given num- ber of factors}

The approach of Section 3 provides neither estimates of the contagion matrix $C$, nor the factor dynamics characterized by $\Phi$ and $\Sigma$, nor filtered factors. We provide 
in this section estimation methods of all parameters, once the number of factors is known.

\subsection{Pseudo-maximum likelihood}

As already noted in Section 2.2 the Gaussian pseudo-likelihood function is easy to compute numerically by applying the linear Kalman filter to the state space representation (2.3)-(2.4). It is also easy to compute numerically the pseudo-maximum likelihood estimates by maximizing this function. To avoid identification problems, this optimization has to be done under the identification restriction:

IR1: $\Omega=I d$,

which is easier to take into account in the optimization problem than the second identification restriction: $B B^{\prime}=I d(\operatorname{IR} 2)$.

\subsection{Asymptotic Least Squares}

An alternative consistent estimation method can be based on the moment restrictions given in Proposition 2 i). Let us for instance consider the two first restrictions written for $h=1,2$. We get:

$$
\left\{\begin{array}{l}
\Gamma_{Y}(1)=C \Gamma_{Y}(0)+B \Phi \operatorname{Cov}(F, Y), \\
\Gamma_{Y}(2)=C^{2} \Gamma_{Y}(0)+\left(C B \Phi+B \Phi^{2}\right) \operatorname{Cov}(F, Y) .
\end{array}\right.
$$

If $\operatorname{Cov}(F, Y)$ is let free, we get $2 n^{2}$ restrictions to find the parameters $C, B, \Phi, \operatorname{Cov}(F, Y)$, that include $n^{2}+n K+K^{2}+n K=(n+K)^{2}$ independent parameters. The order condition for identification is satisfied if:

$$
2 n^{2}>(n+K)^{2} \Longleftrightarrow n^{2}-n K>K^{2} \Longleftrightarrow(n-K)^{2}>2 K^{2} \Longleftrightarrow n \geq(1+\sqrt{2}) K .
$$

Under this order restriction we can apply an asymptotic least-squares approach to 
estimate $C, B, \Phi$, and $\Theta=\operatorname{Cov}(F, Y)$. The ALS estimates are solutions of the optimization problem:

$$
\begin{array}{ll}
\min _{C, B, \Phi, \Theta} \quad \operatorname{Tr}\left\{\left[\hat{\Gamma}_{Y}(1)-C \hat{\Gamma}_{Y}(0)-B \Theta\right]\left[\hat{\Gamma}_{Y}(1)-C \hat{\Gamma}_{Y}(0)-B \Theta\right]^{\prime}\right\} \\
+\operatorname{Tr}\left[\hat{\Gamma}_{Y}(2)-C^{2} \hat{\Gamma}_{Y}(0)-(C B+B \Phi) \Theta\right]\left[\hat{\Gamma}_{Y}(2)-C^{2} \hat{\Gamma}_{Y}(0)-(C B+B \Phi) \Theta\right]^{\prime},
\end{array}
$$

where $\operatorname{Tr}$ denotes the trace operator. The criterion above is written without weighting for expository purpose and $\Theta$ plays the role of a nuisance parameter in the optimization above. Indeed the parameters of interest are the autoregressive parameters $C, B, \Phi$. The last parameter $\Sigma$ can then be deduced from the historical estimate of $V(Y)$ by using the expression of $V(Y)$ given in Lemma 2 in Appendix 2.i).

\subsection{Application to the financial system}

In our application, we focus on 8 of the biggest financial institutions in the euro area, namely: Banco Santander SA, BNP Paribas SA, Commerzbank AG, Crédit Agricole SA, Deutsche Bank AG, Intesa San Paolo SPA, Société Générale SA, and Unicredit SPA. Thus, Germany, France, Italy and Spain are represented in our sample, which allows us to investigate both intra- and international contagion among banks in the euro area.

We convert stock prices and equity values in order to make them comparable with CDS prices, which are quoted in US Dollar. More precisely, we consider the variable $Y_{i, t}$ either as:

- the rate of change in the market capitalization of institution $i$ at time $t$, i.e. $Y_{i, t}=\frac{\operatorname{Cap}_{i, t}-\mathrm{Cap}_{i, t-1}}{\operatorname{Cap}_{i, t-1}}$, where $\mathrm{Cap}_{i, t}$ stands for the market capitalization of institution $i$ in USD at time $t$;

- the variation in the standardized CDS price for institution $i$ at time $t$, i.e. $Y_{i, t}=\Phi^{-1}\left(C D S_{i, t}\right)-\Phi^{-1}\left(C D S_{i, t-1}\right)$;

- the rate of change in the equity value of institution $i$ at time $t$, i .e. $Y_{i, t}=$ $\frac{\mathrm{Equ}_{i, t}-\mathrm{Equ}_{i, t-1}}{\mathrm{Equ}_{i, t-1}}$, where $\mathrm{Equ}_{i, t}$ stands for the book value of institution $i$ in USD 
at time $t$. For this application, we define banks' equity value as their Tier 1 capital $^{8}$.

For each approach, the dependent variable $Y_{t}$ gathers the 8 individual variables $Y_{i, t}$. Data on institutions' market capitalization and CDS come from Bloomberg, while data on the banks' equity value are obtained from Bankscope. Our sample starts in January 2, 2007, and ends on December 20, 2012. In this section, the application builds on quarterly data. An extension to daily data (which are available for CDS prices and market capitalization) is presented in appendix.

\subsubsection{Estimation of the number of factors}

Figure 1 below presents the eigenvalues of the matrix $\hat{C}_{2} S \hat{C}_{2}^{\prime}$ for the 3 datasets $^{9}$
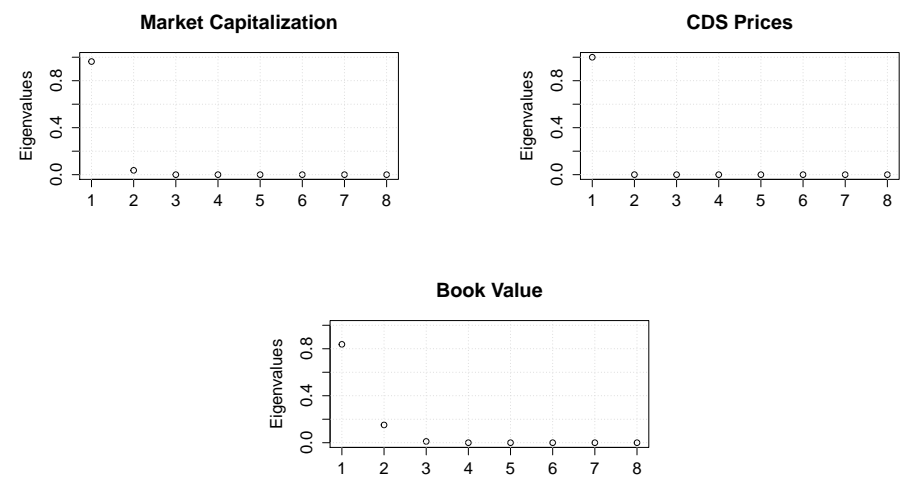

Figure 1: Eigenvalues of the matrix $\hat{C}_{2} S \hat{C}_{2}^{\prime}$ in decreasing order, for the 3 datasets, normalized by the sum of all eigenvalues.

For all datasets, the decrease in the series of eigenvalues shows a clear break after the first eigenvalue, which suggests a number of factor equal to one for all types of data, even if the second eigenvalue is almost significant for the changes in book values.

8"Common equity" would have been closer to the definition of market capitalization; however we preferred to rely on Tier 1 due to data availability constraint.

${ }^{9}$ where $S=\hat{\Gamma}_{Y}(0)-\hat{\Gamma}_{Y}(1) \hat{\Gamma}_{Y}(0)^{-1} \hat{\Gamma}_{Y}(1)^{\prime}$ as in $\left.3 \mathrm{i}\right)$. 


\subsubsection{Estimation of the parameters}

We provide in Table 1 the results of the estimation of the contagion matrix $C$ by Gaussian pseudo-maximum likelihood for the 3 different datasets, setting the number of factors to $K=1$.

We observe a total number of significant connections equal to 42 for the capitalization, 54 for the CDS prices and 50 for the accounting data, to be compared with 64 possible connections. Thus this system of banks is highly interconnected. The rather large number of connections on returns show that both the efficient market hypothesis and the standard two funds theorem are not satisfied. As announced before we have first to focus on the conditional mean before considering conditional variance for financial institutions.

However the different banks do not play the same role. For instance, the return on Commerzbank's stocks does not depend on its lagged return nor the return of the other banks once the effect of the common factor has been taken into account. On the other hand, the Commerzbank has an effect on all the other banks. Another extreme example is the Société Générale, which is affected by all the other banks for the three different series, and aftects all the other banks, except Commerzbank, for the changes in standardized CDS prices and book values. 


\begin{tabular}{|c|c|c|c|c|c|c|c|c|}
\hline & Santander & BNPP & Commerz & CASA & Deutsche & Intesa & SocGen & Unicredit \\
\hline \multirow[t]{3}{*}{ Santander } & -0.569 & -0.165 & 0.081 & 0.250 & 0.437 & 0.065 & 0.045 & -0.111 \\
\hline & -0.349 & 0.006 & -0.091 & -0.237 & -0.111 & 0.643 & -0.150 & 0.315 \\
\hline & -0.042 & 0.336 & 0.110 & -0.193 & -0.160 & 0.300 & 0.113 & 0.642 \\
\hline \multirow[t]{3}{*}{ BNPP } & 0.527 & -0.166 & 0.381 & 0.146 & -0.902 & 0.025 & 0.037 & 0.274 \\
\hline & -0.329 & 0.142 & -0.216 & 0.300 & 0.175 & 0.007 & 0.403 & 0.204 \\
\hline & -0.172 & 0.168 & 0.234 & -0.267 & -0.211 & 0.251 & 0.391 & 0.510 \\
\hline \multirow[t]{3}{*}{ Commerz } & 0.092 & 0.059 & -0.046 & 0.004 & 0.050 & 0.051 & 0.018 & -0.083 \\
\hline & 0.116 & 0.043 & -0.053 & 0.191 & 0.155 & -0.019 & 0.044 & 0.070 \\
\hline & 0.225 & 0.101 & 0.063 & 0.525 & 0.299 & -0.012 & -0.011 & -0.185 \\
\hline \multirow[t]{3}{*}{ CASA } & 0.213 & 0.302 & -0.094 & -0.308 & -0.088 & 0.045 & 0.099 & -0.119 \\
\hline & 0.543 & -0.191 & 0.807 & 0.627 & 0.537 & -0.870 & 0.283 & -0.515 \\
\hline & 0.027 & 0.126 & 0.104 & -0.326 & 0.115 & 0.101 & 0.098 & 0.004 \\
\hline \multirow[t]{3}{*}{ Deutsche } & -0.088 & 0.008 & 0.633 & -0.731 & 0.471 & -0.280 & -0.242 & 0.646 \\
\hline & 0.266 & 0.093 & -0.551 & -0.205 & -0.033 & 0.297 & -0.034 & -0.150 \\
\hline & 0.337 & -0.094 & -0.183 & 0.190 & 0.158 & 0.179 & 0.179 & 0.299 \\
\hline \multirow[t]{3}{*}{ Intesa } & 0.172 & 0.247 & 0.281 & 0.233 & 0.217 & 0.138 & 0.253 & 0.324 \\
\hline & 0.678 & -0.107 & 0.682 & 0.513 & 0.075 & -0.523 & -0.467 & -0.006 \\
\hline & -0.116 & -0.104 & -0.088 & -0.456 & 0.194 & -0.215 & -0.277 & -0.497 \\
\hline \multirow[t]{3}{*}{ SocGen } & -0.336 & -0.245 & -0.267 & 0.526 & 0.292 & 0.142 & 0.202 & -0.195 \\
\hline & -0.186 & 0.062 & -0.103 & -0.526 & -0.412 & 0.636 & -0.370 & 0.620 \\
\hline & -0.303 & -0.135 & -0.299 & 0.454 & -0.204 & -0.365 & -0.289 & -0.118 \\
\hline \multirow[t]{3}{*}{ Unicredit } & 0.109 & 0.199 & -0.038 & 0.144 & -0.139 & 0.105 & 0.069 & -0.186 \\
\hline & -0.410 & 0.134 & -0.286 & -0.273 & 0.036 & 0.277 & 0.558 & -0.206 \\
\hline & -0.024 & -0.127 & -0.227 & 0.191 & 0.386 & 0.056 & 0.213 & -0.139 \\
\hline
\end{tabular}

Table 1: Contagion matrix obtained from banks' market capitalization [1st row in each cell], CDS prices [2rd row] or accounting data [3rd row], at quarterly frequency. Parameters which significantly differ from 0 (at a least $5 \%$ ) are in bold.

The estimated latent factor, or dynamic frailty, is presented in Figure 2 for the three datasets. The three dynamic frailties feature negative autocorrelation (see Table 2) and share a certain degree of commonality, in particular between the market-based frailties. This is formally emphasized in Table 3 and 4, which present the correlations between the three frailties, as well as the factor loadings obtained from a principal component analysis on the 3 factors. Table 4 in particular highlights the existence of a common factor, which loads uniformly on the three frailties and explains about $80 \%$ of their overall variation. The second factor distinguishes market-based indicators from accounting-based one, whereas the last factor mainly discriminates the market 
capitalization frailty from the CDS one. However, we see on Figure 2 that the three factor paths have rather different non-linear features. For instance, the rather large volatilities observed for book values between 2008 and 2009, then after 2011 are difficult to distinguish in the evolutions of factors for stock returns and CDS prices.

\begin{tabular}{cccc} 
& Capitalisation & CDS & Book Value \\
\hline$\phi$ & $-\mathbf{0 . 2 2 3}^{* *}$ & $-\mathbf{0 . 3 7 4}^{* *}$ & $-\mathbf{0 . 6 5 0}^{* *}$ \\
& $(\mathbf{0 . 0 2 1})$ & $(\mathbf{0 . 0 1 5})$ & $(\mathbf{0 . 0 4 1})$
\end{tabular}

Table 2: Autoregressive parameter for the frailty factor $\left({ }^{*}\right.$ significant at $10 \%,{ }^{* *}$ significant at $5 \%)$

\begin{tabular}{l|ccc} 
& Capitalization & CDS & Book Value \\
\hline Capitalization & 1 & 0.661 & 0.450 \\
CDS & 0.661 & 1 & 0.482 \\
Book Value & 0.450 & 0.482 & 1
\end{tabular}

Table 3: Correlation matrix of the dynamic frailty across datasets.

\begin{tabular}{l|ccc} 
& 1st Factor Loading & 2nd Factor Loading & 3rd Factor Loading \\
\hline Capitalization & 0.597 & -0.424 & 0.681 \\
CDS & 0.606 & -0.318 & -0.729 \\
Book Value & 0.525 & 0.848 & 0.067 \\
\hline Proportion of Variation & 0.689 & 0.198 & 0.112
\end{tabular}

Table 4: Factor loadings from the Principal Component Analysis of the estimated frailties. The last line reports the proportion of variation accounted by each factor.

As mentioned before, the factor are defined up to some multiplicative scalar. The factors in Figure 2 have been constructed to be comparable in magnitude (same mean and variance) but also for their interpretation in terms of risk. This is easily seen on Table 6 which provides the factor loading: an increase in the factor value implies a decrease in the stock returns, in CDS prices or book values, respectively. As expected from financial theory the common factor affects all stock returns with the largest influence on the Commerzbank. This compensates the absence of additional effect of the lagged return already discussed.

However the interpretation of the frailty as a common factor is no longer valid when we consider the analysis based on either CDS price or book values, since in both 
cases only one beta is significant for BNPP and Commerzbank, respectively. The interpretation of the significant beta is now different. If all betas were equal to 0 , the dynamic model would be a $\operatorname{VAR}(1)$ model, with short memory features. The presence of significant beta introduces longer memory, and this longer memory is channeled through a single bank (either Commerzbank or BNPP).
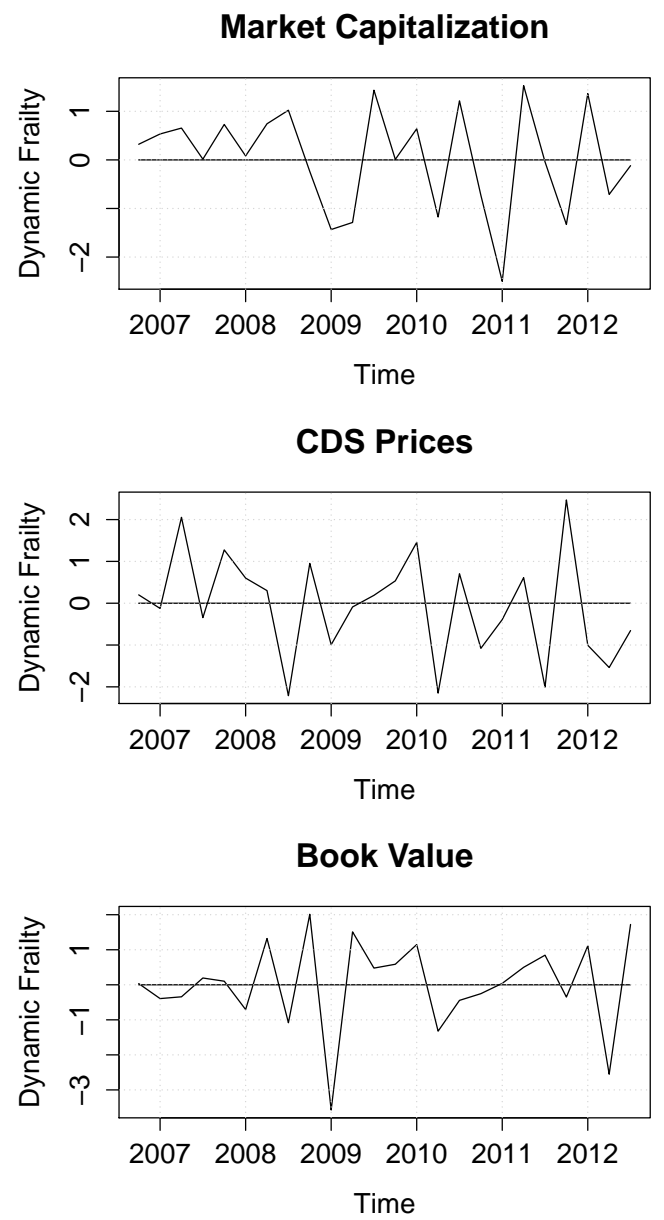

Figure 2: Estimated dynamic frailties obtained from banks' market capitalization (top), CDS prices (middle), book values (bottom), at quarterly frequency. 


\begin{tabular}{lcccccccc} 
& Santander & BNPP & Commerz & CASA & Deutsche & Intesa & SocGen & Unicredit \\
\hline Capitalization & $-\mathbf{0 . 1 5 3 0}^{* *}$ & $-\mathbf{0 . 2 1 6}^{*}$ & $-\mathbf{0 . 3 3 5}^{* *}$ & $-\mathbf{0 . 2 1 6}^{* *}$ & $-\mathbf{0 . 1 9 0}^{* *}$ & $-\mathbf{0 . 1 6 0}^{* *}$ & $-\mathbf{0 . 2 0 4}^{* *}$ & $-\mathbf{0 . 2 0 8}^{* *}$ \\
& $(\mathbf{0 . 0 4 9 )}$ & $\left(\mathbf{0 . 1 1 6}^{*}\right)$ & $(\mathbf{0 . 1 0 6})$ & $(\mathbf{0 . 0 7 5})$ & $(\mathbf{0 . 0 6 4})$ & $(\mathbf{0 . 0 7 1})$ & $(\mathbf{0 . 0 5 1})$ & $(\mathbf{0 . 0 7 1})$ \\
CDS & -0.046 & $-\mathbf{0 . 0 4 3}^{* *}$ & -0.041 & -0.052 & -0.038 & -0.027 & -0.047 & -0.023 \\
\multirow{3}{*}{ Book Value } & $(0.032)$ & $(\mathbf{0 . 0 2 2})$ & $(0.041)$ & $(0.035)$ & $(0.024)$ & $(0.075)$ & $(0.0786)$ & $(0.037)$ \\
& -0.029 & -0.065 & $-\mathbf{0 . 0 8 4}^{*}$ & -0.091 & -0.026 & -0.042 & -0.037 & -0.028 \\
& $(0.031)$ & $(0.044)$ & $\mathbf{( 0 . 0 4 6 )}$ & $(0.090)$ & $(0.038)$ & $(0.051)$ & $(0.052)$ & $(0.031)$
\end{tabular}

Table 6: Matrix of loadings to the frailty factor obtained from banks' market capitalization, CDS prices or accounting data $\left({ }^{*}\right.$ significant at $10 \%,{ }^{* *}$ significant at $\left.5 \%\right)$.

\section{Concluding remarks}

The aim of this paper is to explain how to disentangle the direct and indirect (contagion) effects of the exogenous shocks on the financial situations of the banks or insurance companies. For this purpose we have considered a linear dynamic model with both common frailty and autoregressive feature, and introduce an appropriate methodology to estimate the contagion matrix, the sensitivity of institutions to the factors and to reconstitute the underlying factor paths.

Our method has been applied to a set of eight banks and different measures of their financial position, measured by the change in their market capitalization, CDS prices or book values, respectively. Even if we might expect similar results for the three different measures, the analysis shows that the revealed interconnections are significantly different, in particular between market and accounting data.

In practice, the European banking system contains much more than 8 banks and the extension of the methodology will require methods for large scale factor models based on sparse estimator or Lasso (see e.g. Barigozzi, Brownlees (2013) for such an approach in a model without frailty). 


\section{R E F E R E N C E S}

Allen, F., and D., Gale (2000) : "Financial Contagion", Journal of Political Economy, 108, 1-33.

Barigozzi, M., and C., Brownlees (2013) : "Nets: Network Estimation for Time Series", DP Univ. of Pompeu Fabra.

Beine, M., and A., Hecq (1999) : "Inference in Codependence : Some Monte Carlo Results and Applications", Annales d'Economie et de Statistique, 54, 69-90.

Bekaert, G., Harvey, C., and A., Ng (2003)) : "Market Integration and Contagion", Journal of Business, 78, 39-70.

Billio, M., Caporin, M., Pelizzon, L., and D., Sartore (2012) : "CDS Industrial Sector Indices, Credit and Liquidity Risk", DP Univ. of Venice.

Billio, M., Getmansky, M., Lo, A., and L., Pelizzon (2012) : "Econometric Measures of Correlation and Systematic Risk in the Finance and Insurance Sectors", Journal of Financial Economics, 104, 535-539.

Brownlees, C., and R., Engle (2012) : "Volatility, Correlation and Tails for Systemic Risk Measurement", NYU DP.

Candelon, B., and A., Hecq (2000) : "Stability of Okun's Law in a Codependent System", Applied Economic Letters, 7, 687-693.

Darolles, S., Gagliardini, P., and C., Gourieroux (2013) : "Survivor of Hedge Funds: Frailty vs Contagion", CREST DP. 
Degerine, S. (1990) : "Canonical Partial Autocorrelation Function of Multivariate Time Series", The Annals of Statistics, 18, 961-971.

Degerine, S. (1994) : "Sample Partial Autocorrelation Function of a Multivariate Time Series", Journal of Multivariate Analysis, 50, 294-313.

Delatte, A.L, Gex, M., and A., Lopez-Villavencio (2012) : "Has the CDS Market Influenced the Borrowing Cost of European Countries During the Sovereign Crisis?", Journal of International Money and Finance, 31, 481-497.

Diebold, F., and M., Nerlove (1989) : "The Dynamics of Exchange Rate Volatility: A Multivariate Latent Factor ARCH Model", Journal of Applied Econometrics, 4, 1-21.

Duffie, D., Eckner, A., Horel, G., and L. Saita (2009) : "Frailty Correlated Default", Journal of Finance, 64, 2089-2123.

Dungey, M., and V., Martin (2003) : "Equity Transmission Mechanisms from Asia To Australia: Interdependence or Contagion?", Australian Journal of Management, $28,157-182$.

Dungey, M., Fry, R., Gonzalez-Hermosillo, B., and V., Martin (2005) : "Empirical Modelling of Contagions : A Review of Methodologies", Quantitative Finance, 5, $9-24$.

Engle, R., and S., Kozicki (1993) : "Testing for Common Features", Journal of Business and Economic Statistics, 11, 369-380.

Financial Stability Board (2009) : "Guidance to Assess the Systemic Importance of Financial Institutions, Markets and Instruments", Initial Considerations" 
Fisher, R. (1924) : "The Distribution of the Partial Correlation Coefficient", Metron, 3, 329-332.

Forbes, R., and R., Rigobon (2002) : "No Contagion, Only Interdependence : Measuring Stock Market Comovements", Journal of Finance, 57, 2223-2261.

Forni, M., Hallen, N., Lippi, M., and L., Reichlin (2000) : "The Generalized Dynamic Factor Model: Identification and Estimation", The Review of Economics and Statistics, 83, 540-554.

Gourieroux, C., and A., Monfort (1997) : "Time Series and Dynamic Models", Cambridge University Press, 670p.

Gourieroux, C., Monfort, A., and E., Renault (1993) : "Test sur le noyau, l'image et le rang de la matrice des coefficients d'un modèle linéaire multivarié", Annales d'Economie et de Statistique, 32, 81-111

Gourieroux, C., and I., Peaucelle (1983) : "Detecting a Long Run Relationship", Canadian Journal of Economics.

Gourieroux, C., and I., Peaucelle (1993) : "Series codépendantes : application à l'hypothèse de parité du pouvoir d'achat", in Macroéconomie, développements récents, ed. Economica, 285-306.

Hautsch, N., Schaumburg, J., and M., Schueule (2012) : "Financial Network Systemic Risk Contributions", DP 2012-053, Humboldt University.

King, M., and S., Wadhwani (1990) : "Transmission of Volatility Between Stock Markets", Review of Financial Studies, 3, 5-33.

Kugler, P., and P., Schwendener (1992) : "Codependence in a VAR Framework", Bern Univ. DP. 
Kugler, P., and K., Neusser (1993) : "International Real Interest Rate Equalization: A Multivariate Time Series Approach", Journal of Applied Econometrics, 8, 163-174.

Merton, R. (1974) : "On the Pricing of Corporate Debt: The Risk Structure of Interest Rates", Journal of Finance, 29, 449-470.

Muirhead, R. (1982) : "Aspects of Multivariate Statistical Theory", Wiley, NewYork.

Pindyck, R., and J., Rotemberg (1990) : "The Excess co-Movement of Commodity Prices", The Economic Journal, 100, 1173-1189.

Pindyck, R., and J., Rotemberg (1993) : "The Comovement of Stock Prices", The Quartely Journal of Economics, 108, 1073-1104.

Ramsey, F. (1974) : "Characterization of the Partial Autocorrelation Function", Ann. Statist., 2, 1296-1301.

Reinsel, G. (1993) : "Elements of Multivariate Time Series Analysis", Springer Verlag, Berlin.

Tiao, G., and R., Tsay (1985) : "A Canonical Correlation Approach to Modeling Multivariate Time Series", in Proceedings of the Business and Economic Statistic Section of the American Statistical Association, Washington DC, 112-120..

Vahid, F., and R., Engle (1997) : "Codependent Cycles", Journal of Econometrics, 80, 199-221.

Velu, P., Reinsel, G., and D., Wichern (1986) : "Reduced Rank Models for Multivariate Time Series", Biometrika, 73, 105-118. 
Appendix 1

\section{Review of the literature}

\begin{tabular}{|c|c|c|c|c|c|}
\hline Authors & data & $\begin{array}{l}\text { observ. } \\
\text { factor }\end{array}$ & $\begin{array}{l}\text { unobserv. } \\
\text { factor }\end{array}$ & contagion & $\begin{array}{c}\text { linear } \\
\text { vs nonlinear }\end{array}$ \\
\hline $\begin{array}{l}\text { Diebold, } \\
\text { Nerlove (1989) }\end{array}$ & XR & & X & & $\mathrm{ARCH}$ \\
\hline $\begin{array}{l}\text { King, } \\
\text { Wadhwani (1990) }\end{array}$ & I & & $\mathrm{X}$ & & $\mathrm{L}$ \\
\hline $\begin{array}{l}\text { Forbes, } \\
\text { Rigobon (2002) }\end{array}$ & I & & Xs & Xs & L \\
\hline $\begin{array}{l}\text { Dungey, } \\
\text { Martin (2003) }\end{array}$ & S & & Xs & Xs & L \\
\hline $\begin{array}{l}\text { Dungey et al. } \\
(2005)\end{array}$ & S & & X & Xs & L \\
\hline $\begin{array}{l}\text { Brownlees, } \\
\text { Engle(2012) }\end{array}$ & S & & & X & $\mathrm{ARCH}$ \\
\hline $\begin{array}{l}\text { Billio et al. } \\
(2012)\end{array}$ & & & & X & \\
\hline $\begin{array}{l}\text { Darolles, Gagliardini, } \\
\text { Gourieroux (2012) }\end{array}$ & $\mathrm{HF}$ & & X & X & $\begin{array}{l}\text { NL (Poisson model with } \\
\text { stochastic intensity) }\end{array}$ \\
\hline $\begin{array}{l}\text { Barigozzi, } \\
\text { Brownlees (2013) }\end{array}$ & $\mathrm{S}$ & X & & X & $\mathrm{L}$ \\
\hline
\end{tabular}

Data: BS (balance sheet), S (stock), I (Market indexes), XR (exchange rates), B (bond), CDS, HF (hedge fund).

Linear VS Nonlinear model: L (linear model), ARCH (ARCH model), NL (nonlinear model different from ARCH).

Cross (X): Yes, no cross: No 
Contagion: indexed by $s$, when contagion arises at the same date, that is simultaneously.

Unobserved factor: indexed by $s$ for the state factor. 
Appendix 2

\section{Second-order properties}

By considering the VaR dynamic of process $\left(Z_{t}\right)$, we get:

$$
\begin{cases}E\left(Z_{t}\right) & =0 \\ V\left(Z_{t}\right) & =\Gamma_{Z}(0)=V(w)+\Psi V(w) \Psi^{\prime}+\ldots+\Psi^{h} V(w) \Psi^{h^{\prime}}+\ldots \\ \operatorname{Cov}\left(Z_{t}, Z_{t-h}\right) & =\Gamma_{Z}(h)=\Psi^{h} \Gamma_{Z}(0), h \geq 0 .\end{cases}
$$

These expressions can be used to derive the second-order properties of the observable process $\left(Y_{t}\right)$.

Lemma 1: We have:

$$
\Psi^{h}=\left(\begin{array}{cc}
C^{h} & D_{h} \\
0 & \Phi^{h}
\end{array}\right)
$$

where $D_{h}=C D_{h-1}+B \Phi^{h}, h \geq 1, D_{0}=0$, or equivalently $D_{h}=\sum_{k=0}^{h-1}\left(C^{k} B \Phi^{h-k}\right)$.

Proof: Since:

$$
\Psi^{h}=\Psi \Psi^{h-1}=\left(\begin{array}{cc}
C & B \Phi \\
0 & \Phi
\end{array}\right)\left(\begin{array}{cc}
C^{h-1} & D_{h-1} \\
0 & \Phi^{h-1}
\end{array}\right)
$$

we deduce $D_{h}=C D_{h-1}+B \Phi^{h}$.

Lemma 2: We have:

$$
V(Z)=\left[\begin{array}{cc}
V(Y) & \operatorname{Cov}(Y, F) \\
\operatorname{Cov}(F, Y) & V(F)
\end{array}\right]
$$

where: 


$$
\begin{gathered}
V(F)=\Phi V(F) \Phi^{\prime}+\Omega=\sum_{h=0}^{\infty} \Phi^{h} \Omega \Phi^{h^{\prime}}, \\
\operatorname{Cov}(Y, F)=\operatorname{CCov}(Y, F) \Phi^{\prime}+B \Phi V(F) \Phi^{\prime}+B \Omega, \\
V(Y)=\sum_{h=0}^{\infty} C^{h} \Delta C^{h^{\prime}},
\end{gathered}
$$

with

$$
\Delta=B \Phi \operatorname{Cov}(F, Y) C^{\prime}+C \operatorname{Cov}(Y, F) \Phi^{\prime} B^{\prime}+B \Phi V(F) \Phi^{\prime} B^{\prime}+\Sigma+B \Omega B^{\prime} .
$$

Proof: We have:

$$
\begin{aligned}
\Gamma_{Z}(0) & =\Psi \Gamma_{Z}(0) \Psi^{\prime}+V w \\
& =\left(\begin{array}{cc}
C & B \Phi \\
0 & \Phi
\end{array}\right)\left(\begin{array}{cc}
V(Y) & \operatorname{Cov}(Y, F) \\
\operatorname{Cov}(F, Y) & V(F)
\end{array}\right)\left(\begin{array}{cc}
C^{\prime} & 0 \\
\Phi^{\prime} B^{\prime} & \Phi^{\prime}
\end{array}\right) \\
& +\left(\begin{array}{cc}
\Sigma+B \Omega B^{\prime} & B \Omega \\
\Omega B^{\prime} & \Omega
\end{array}\right) .
\end{aligned}
$$

By identification we deduce the recursive system:

$$
\begin{aligned}
V(F) & =\Phi V(F) \Phi^{\prime}+\Omega \\
\operatorname{Cov}(Y, F) & =C \operatorname{Cov}(Y, F) \Phi^{\prime}+B \Phi V(F) \Phi^{\prime}+B \Omega, \\
V(Y) & =C V(Y) C^{\prime}+B \Phi \operatorname{Cov}(F, Y) C^{\prime}+C \operatorname{Cov}(Y, F) \Phi^{\prime} B^{\prime} \\
& +B \Phi V(F) \Phi^{\prime} B^{\prime}+\Sigma+B \Omega B^{\prime}
\end{aligned}
$$


These lemmas are used to deduce equations satisfied by the autocovariance function of the observable process $\left(Y_{t}\right)$. Indeed we have:

$$
\operatorname{Cov}\left(Z_{t}, Z_{t-h}\right)=\Psi^{h} \Gamma_{Z}(0)=\left(\begin{array}{cc}
C^{h} & D_{h} \\
& \\
0 & \Phi^{h}
\end{array}\right)\left(\begin{array}{cc}
V(Y) & \operatorname{Cov}(Y, F) \\
\operatorname{Cov}(F, Y) & V(F)
\end{array}\right)
$$

By considering the first block diagonal element of this product, we get the recursion in Proposition 2. 


\section{Appendix 3}

\section{Application to daily data}

In this appendix, we extend the analysis conducted in Section 4.3 to daily data, building on banks' market capitalization and CDS prices which are available at daily frequency. For this application, we use the same dataset as in Section 4.3: data come from Bloomberg, our sample starts in January 2, 2007 and ends in December 20, 2012.

\begin{tabular}{lcccccccc} 
& Santander & BNPP & Commerz & CASA & Deutsche & Intesa & SocGen & Unicredit \\
\hline Santander & 0,024 & $-0,008$ & $-0,082$ & 0,037 & $-0,007$ & $\mathbf{0 , 1 2 8 ^ { * * }}$ & 0,021 & 0,050 \\
& $(0,054)$ & $(0,064)$ & $(0,098)$ & $(0,072)$ & $(0,057)$ & $(\mathbf{0 , 0 5 6})$ & $(0,067)$ & $(0,070)$ \\
BNPP & $-0,022$ & 0,004 & $-0,120$ & $-0,021$ & 0,029 & $-0,088$ & $-0,098$ & $-0,053$ \\
& $(0,066)$ & $(0,060)$ & $(0,092)$ & $(0,065)$ & $(0,057)$ & $(0,059)$ & $(0,063)$ & $(0,066)$ \\
CASA & $-0,006$ & 0,014 & 0,007 & 0,015 & $-0,003$ & $-0,011$ & 0,014 & 0,000 \\
& $(0,035)$ & $(0,040)$ & $(0,051)$ & $(0,045)$ & $(0,041)$ & $(0,037)$ & $(0,047)$ & $(0,045)$ \\
Commerz & 0,010 & 0,012 & 0,012 & $-0,041$ & $-0,005$ & 0,004 & 0,006 & 0,064 \\
& $(0,054)$ & $(0,062)$ & $(0,094)$ & $(0,060)$ & $(0,054)$ & $(0,061)$ & $(0,063)$ & $(0,067)$ \\
Deustche & $-0,006$ & $-0,021$ & 0,011 & 0,049 & 0,039 & 0,003 & $\mathbf{0 , 1 3 0}$ & 0,028 \\
& $(0,048)$ & $(0,058)$ & $(0,077)$ & $(0,058)$ & $(0,049)$ & $(0,054)$ & $(\mathbf{0 , 0 4 8})$ & $(0,057)$ \\
Intesa & 0,042 & 0,019 & $\mathbf{0 , 4 0 6} \mathbf{n}^{* *}$ & 0,086 & $\mathbf{0 , 1 6 8}$ & $-0,019$ & 0,058 & $-0,012$ \\
& $(0,057)$ & $(0,059)$ & $(\mathbf{0 , 0 5 9})$ & $(0,067)$ & $(\mathbf{0 , 0 5 3})$ & $(0,055)$ & $(0,063)$ & $(0,065)$ \\
SocGen & $-0,054$ & $-0,027$ & $-0,058$ & $-0,014$ & $-\mathbf{0 , 1 3 1}$ & $-0,018$ & $-0,031$ & $-0,049$ \\
& $(0,057)$ & $(0,053)$ & $(0,071)$ & $(0,058)$ & $(\mathbf{0 , 0 4 3})$ & $(0,053)$ & $(0,055)$ & $(0,058)$ \\
Unicredit & 0,043 & 0,058 & $-0,045$ & 0,005 & 0,005 & 0,054 & 0,053 & 0,024 \\
& $(0,044)$ & $(0,054)$ & $(0,056)$ & $(0,056)$ & $(0,044)$ & $(0,049)$ & $(0,057)$ & $(0,050)$
\end{tabular}

Table 7: Contagion matrix obtained from market capitalization $\left({ }^{* *}\right.$ significant at $5 \%$, at daily 


\begin{tabular}{lcccccccc} 
& Santander & BNPP & Commerz & CASA & Deutsche & Intesa & SocGen & Unicredit \\
\hline Santander & 0,062 & 0,033 & 0,034 & 0,061 & 0,043 & $\mathbf{0 , 1 4 5}$ & 0,033 & 0,073 \\
& $(0,066)$ & $(0,064)$ & $(0,059)$ & $(0,066)$ & $(0,057)$ & $(\mathbf{0 , 0 7 3})$ & $(0,064)$ & $(0,075)$ \\
BNPP & $-0,042$ & $-0,069$ & 0,017 & $-0,006$ & 0,009 & $-0,049$ & 0,023 & $-0,008$ \\
& $(0,083)$ & $(0,080)$ & $(0,077)$ & $(0,078)$ & $(0,070)$ & $(0,091)$ & $(0,081)$ & $(0,091)$ \\
Commerz & $-0,038$ & 0,003 & 0,057 & 0,029 & 0,013 & $-0,024$ & 0,086 & $-0,022)$ \\
& $(0,087)$ & $(0,080)$ & $(0,073)$ & $(0,081)$ & $(0,071)$ & $(0,090)$ & $(0,085)$ & $(0,094)$ \\
CASA & 0,016 & 0,082 & 0,042 & $-0,023$ & 0,001 & 0,019 & $\mathbf{0 , 1 2 9} *$ & 0,019 \\
& $(0,075)$ & $(0,076)$ & $(0,063)$ & $(0,071)$ & $(0,069)$ & $(0,082)$ & $(\mathbf{0 , 0 7 1})$ & $(0,080)$ \\
Deutsche & $-0,010$ & 0,029 & $-0,049$ & $-0,028$ & $-0,010$ & $-0,069$ & $-\mathbf{0 , 1 3 2}$ & $-0,059$ \\
& $(0,074)$ & $(0,066)$ & $(0,068)$ & $(0,070)$ & $(0,062)$ & $(0,080)$ & $(\mathbf{0 , 0 7 4})$ & $(0,080)$ \\
Intesa & 0,085 & 0,021 & 0,003 & 0,071 & 0,025 & $-0,138$ & 0,050 & 0,095 \\
& $(0,116)$ & $(0,099)$ & $(0,095)$ & $(0,104)$ & $(0,089)$ & $(0,125)$ & $(0,107)$ & $(0,129)$ \\
SocGen & $0,039)$ & $0,037)$ & $0,026)$ & $0,118)$ & $0,076)$ & $0,097)$ & $-0,017)$ & 0,042 \\
& $(0,088)$ & $(0,076)$ & $(0,075)$ & $(0,080)$ & $(0,072)$ & $(0,092)$ & $(0,078)$ & $(0,090)$ \\
Unicredit & $-0,019)$ & $-0,031)$ & $0,013)$ & $-0,080)$ & $-0,021)$ & $0,155)$ & $-0,025)$ & 0,024 \\
& $(0,104)$ & $(0,095)$ & $(0,089)$ & $(0,093)$ & $(0,082)$ & $(0,114)$ & $(0,094)$ & $(0,115)$
\end{tabular}

Table 8: Contagion matrix obtained from CDS prices $\left({ }^{*}\right.$ significant at $10 \%,{ }^{* *}$ significant at $5 \%$ ), at daily frequency.

As expected, we get a smaller number of interconnections, that is a total of 5 for market capitalization and 3 for the CDS prices. These small numbers of connections can provide much larger numbers when the frequency of the observations diminishes, due to the effect of time aggregation on the contagion matrix discussed in Section 2.5, that is the transformation $C \rightarrow C^{h}=C^{60}$.

\begin{tabular}{ccc} 
& Capitalisation & CDS \\
\hline$\phi$ & -0.013 & 0.138 \\
& $(0.215)$ & $(0.381)$
\end{tabular}

Table 9: Autoregressive parameter for the frailty factor

\begin{tabular}{l|cc} 
& Capitalization & CDS \\
\hline Capitalization & 1 & 0.61 \\
CDS & 0.61 & 1
\end{tabular}

Table 10: Correlation matrix of the dynamic frailty across datasets. 


\begin{tabular}{l|cc} 
& 1st Factor Loading & 2nd Factor Loading \\
\hline Capitalization & 0.707 & -0.707 \\
CDS & 0.707 & 0.707 \\
\hline Proportion of Variance & 0.81 & 0.19
\end{tabular}

Table 11: Factor loadings .
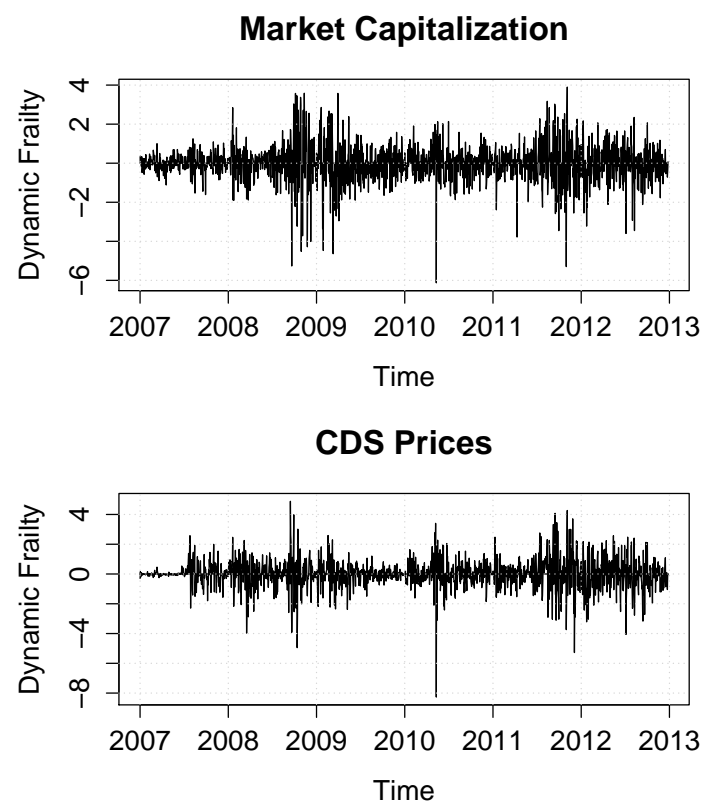

Figure 3: Estimated dynamic frailties obtained from banks' market capitalization (top), CDS prices (bottom), at daily frequency.

\begin{tabular}{lcccccccc} 
& Santander & BNPP & Commerz & CASA & Deutsche & Intesa & SocGen & Unicredit \\
\hline Capitalization & $\mathbf{2 . 5 2 5}^{* *}$ & $-\mathbf{3 . 1 0 2}^{* *}$ & $-\mathbf{3 . 5 4 1}^{* *}$ & $-\mathbf{3 . 2 9 0}^{* *}$ & $-\mathbf{3 . 0 6 5}^{* *}$ & $-\mathbf{2 . 9 4 9}^{* *}$ & $-\mathbf{3 . 3 4 9}^{* *}$ & $-\mathbf{3 . 4 1 9}^{* *}$ \\
& $(\mathbf{0 . 0 6 2})$ & $(\mathbf{0 . 0 5 9})$ & $(\mathbf{0 . 1 1 1})$ & $(\mathbf{0 . 0 6 4})$ & $(\mathbf{0 . 0 5 5})$ & $(\mathbf{0 . 0 5 3})$ & $(\mathbf{0 . 0 5 9})$ & $(\mathbf{0 . 0 7 2})$ \\
CDS & $-\mathbf{1 . 5 6 7}^{* *}$ & $-\mathbf{1 . 3 7 9}^{* *}$ & $-\mathbf{1 . 3 3 0}^{* *}$ & $-\mathbf{1 . 4 2 7}^{* *}$ & $-\mathbf{1 . 2 5 6}^{* *}$ & $-\mathbf{1 . 6 7 2}^{* *}$ & $-\mathbf{1 . 4 7 2}^{* *}$ & $-\mathbf{1 . 7 2 8}^{* *}$ \\
& $(\mathbf{0 . 0 3 0})$ & $(\mathbf{0 . 1 4 7})$ & $(\mathbf{0 . 3 1 8})$ & $(\mathbf{0 . 1 9 0})$ & $(\mathbf{0 . 2 5 6})$ & $(\mathbf{0 . 4 5 0})$ & $(\mathbf{0 . 2 0 4})$ & $(\mathbf{0 . 2 9 8})$
\end{tabular}

Table 12: Loadings to the frailty factor $\left({ }^{*}\right.$ significant at $10 \%,{ }^{* *}$ significant at $\left.5 \%\right)$

At the daily level, we get rally common factors for the wo cases. As in the case of the quarterly analysis these factors show a significantly different dynamic. In particular 
the factor for the capitalization features more important periods of high volatility both for the magnitude of the volatility and the length of the period, especially during the 2008 financial crisis. 